\title{
THE CORNELL ERL SUPERCONDUCTING 2-CELL INJECTOR CAVITY STRING AND TEST CRYOMODULE*
}

\author{
M. Liepe ${ }^{\dagger}$, S. Belomestnykh, E. Chojnacki, V. Medjidzade, H. Padamsee, P. Quigley, J. Sears, \\ V. Shemelin, V. Veshcherevich, CLASSE, Cornell University, Ithaca, NY, USA
}

\begin{abstract}
Cornell University is developing and fabricating a SRF injector cryomodule for the acceleration of the high current (100 mA) beam in the Cornell ERL prototype and ERL light source. Major challenges include emittance preservation of the low energy, ultra low emittance beam, cw cavity operation, and strong HOM damping with efficient HOM power extraction. Prototypes have been completed for the 2-cell niobium cavity with helium vessel, coaxial blade tuner with piezo fine tuners, twin high power input couplers, and beam line HOM absorbers loaded with ferrites and ceramics. Axial symmetry of HOM absorbers, together with two symmetrically placed input couplers per cavity, avoids transverse on-axis fields, which would cause emittance growth. A one-cavity cryostat has been designed following concepts of the TTF cryostat, and is presently under fabrication and assembly. The cryostat design has been optimized for precise cavity alignment, good magnetic shielding, and high dynamic cryogenic loads from the RF cavities, input couplers, and HOM loads. In this paper we report on the status of the assembly and first test of the one-cavity test cryostat.
\end{abstract}

\section{INTRODUCTION}

Cornell University's Laboratory for Accelerator based Sciences and Education (CLASSE) is exploring the potential of a X-ray light source based on the Energy-RecoveryLinac (ERL) principle [1]. This type of light source promises superior X-ray performance as compared to conventional third generation light sources [2], but several accelerator physics and technology challenges need to be addressed before a full energy ERL light source can be built. These challenges result primarily from the high current, ultra low emittance beam required at the undulator locations beyond the main linac. As a first and crucial step, Cornell has launched an extensive R\&D program to study and demonstrate the production and preservation of the ultralow emittance beam in the ERL injector. A prototype of the injector is presently under construction at Cornell, and first beam in the full injector is expected for 2008 [3].

One of the most challenging components in the injector is its cryomodule hosting five superconducting (SC) 2-cell cavities. In $\mathrm{CW}$ operation, the cavities not only have to transfer a total power of $500 \mathrm{~kW}$ to the beam, but also have to do so without destroying its ultra-low emittance. These requirements together with a high beam current of

\footnotetext{
* Work supported by the National Science Foundation.

†MUL2@cornell.edu
}

up to $100 \mathrm{~mA}$ and short bunch lengths of less then 600 $\mu m$ put high demands on the superconducting cavities, the Higher-Order-Mode (HOM) damping scheme, the RF input couplers, and the cryovessel itself. Solutions to all these challenges have been found [4], and prototypes of all beam line components (SRF cavities, HOM loads, input couplers, and frequency tuners) have been fabricated and tested individually. A one cavity horizontal test cryostat (HTC) is presently under assembly at Cornell. The HTC follows the same design as the full injector cryostat, but hosts one cavity with two HOM loads only instead of five cavities with six HOM loads. A first test of the HTC is scheduled for July 2007, and will give a full system test of all beam line components as well as a first test of the new cryomodule design realized in the ERL injector.

In the following we report on the status of the beam line prototypes and the HTC assembly and impressions from this first assembly, as well as discuss test plans and future work.

\section{HTC BEAM LINE}

Prototypes of the beam line components have been fabricated, and tested individually. The HTC beam line string consists out of the following main components:

Cavity: A prototype $1.3 \mathrm{GHz} 2$-cell ERL injector cavity [5] has been fabricated in house, tested vertically and equipped with a He-vessel. After the successful vertical test of the first prototype, five more 2-cell cavities have been built and tested vertically at Cornell. Refer to [6] for details of the cavity fabrication and test. All cavities performed satisfactory in vertical acceptance tests. A processable multipacting barrier was found in the large beam pipe, in correspondence with multipacting simulations [6] .

HOM Loads: Two Cornell ERL prototype HOM loads have been fabricated at Cornell and will be used in the first HTC test [7]. A combination of three different RF absorbing materials (two ferrite types and one ceramic) is used to guarantee efficient RF absorption starting from low frequency to $100 \mathrm{GHz}$, as required by the high beam current and short bunch length [8]. Reliable parameters have been developed for the soldering and brazing of the absorber materials to thermally matched metals. Additional HOM loads for the full injector cryomodule have been ordered from industry.

Input Couplers: Two prototype Cornell ERL CW 1.3 GHz input couplers have been fabricated by industry and have been high power tested at Cornell. The specified maximum RF power of $50 \mathrm{~kW}$ was reached in $\mathrm{CW}$ operation, but insufficient cooling of some coupler sections was found 
[9]. The coupler design was modified to increase cooling of these sections, and additional couplers are presently under fabrication in industry.

Frequency Tuner: The frequency tuner for the 2-cell ERL injector cavities has been adopted from the DESY/INFN blade tuner design [10]. Short piezo-electric actuators have been integrated in the frequency tuner mechanism to allow for fast microphonics compensation. In addition, the tuner mount has been redesigned for easy exchange of the motor/harmonic drive assembly while the cavity string is installed in the vacuum vessel. In the Cornell ERL cryomodule, the tuner motor can be accessed through ports in the vacuum vessel and shields to allow for replacement in case of a motor failure. Six blade tuners have been fabricated by industry.

The beam line string for the horizontal test cryomodule has been assembled in Cornell's class 100 clean room, using a special support fixture to align the beam line components for easy flange connection. The finished cavity string is shown in Figure 1.

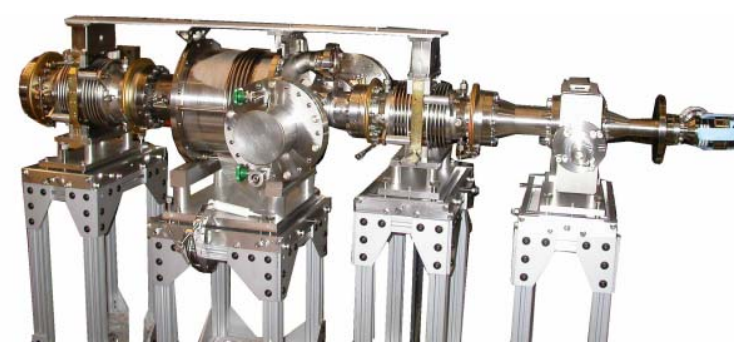

Figure 1: HTC beam line string

\section{CRYOMODULE DESIGN}

The ERL injector module and the horizontal test module design is based on the TTF cryomodule [11], with beam line components supported from a large diameter helium gas return pipe and all cryogenic piping located inside the module. Although this cryomodule concept has primarily been optimized for longer superconducting linacs, it has been chosen here for the short injector SRF section so that it can also serve as a prototype for ERL main linac cryomodule. The TTF type module design was significantly redesigned to fulfill ERL specific requirements (see also Figure 2 and Table 1):

- CW cavity operation results in high $2 \mathrm{~K}$ cryogenic loads of up to $5 \mathrm{~W}$ per 2-cell cavity. The $2 \mathrm{~K}$-2-phase line diameter has been increased to $10 \mathrm{~cm}$ to support high loads.

- A high cavity quality factor $Q_{0}$ is important for efficient CW cavity operation. Three layers of magnetic shield (two around the individual cavities and a third on top of the $80 \mathrm{~K}$ thermal shield) give effective shielding of external magnetic fields to support high $Q_{0}$ cavity operation.

07 Accelerator Technology Main Systems
- The power dissipation at the cold HOM loads and the cold part of the high power input couplers can not be intercepted by thermal conduction through copper braids. Direct gas cooling of chosen $5 \mathrm{~K}$ and $80 \mathrm{~K}$ intercept points with He-gas flow through small heat exchangers is used in the Cornell ERL cryomodule. The $5 \mathrm{~K}$ and $80 \mathrm{~K}$ He-gas is supplied via large pipes and distributed to the individual heat exchangers at the HOM loads and input couplers through 1/4" tubing.

- The cryomodule design has been simplified by using only one layer of thermal shield (at $80 \mathrm{~K}$ ). A 5K shield is of smaller importance in CW SRF cryomodules, since the high dynamic loads dominate over static loads.

- The cavities and HOM loads are supported via fixed (i.e. non-sliding) supports to Helium-Gas-ReturnPipe (HGRP) sections made out of Titanium. The HGRP sections are connected by bellows. The fixed support uses precise machined surfaces and alignment fiducials. This does not only simplify attaching the beam line to the HGRP, but also makes any final cavity alignment at this stage obsolete, resulting in a stiff and well aligned cavity string.

- The alignment bolts at the HGRP support posts are accessible even while the module is cold. This allows for beam based fine alignment of the cavities, which are mounted to the short HGRP sections, while the cryomodule is cold.

- New short module end sections without He reservoir give short cold-warm transitions at the module ends. This is essential especially in the ERL injector, where the distance between the photo-emission DC gun and the first SRF cavity needs to be minimized. The beam line has one gate-valve on each module end, which is located inside of the module with its drive unit outside of the module. This way no external gate vales are required at the module ends.

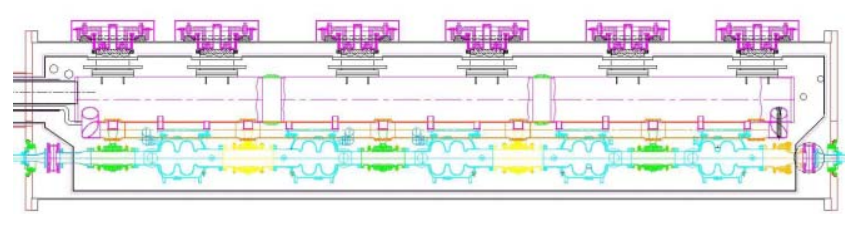

Figure 2: 2D cross section of the ERL injector module.

\section{TEST CRYOMODULE ASSEMBLY}

At the time of writing, the assembly of the ERL horizontal test cryomodule is nearly finished; see Figure 3. Extensive diagnostics have been installed for the first test [12]. The overall design concept has shown to allow for a fast and easy assembly. The beam line vacuum is maintained after a bake throughout the entire assembly process. A final warm-coupler in-situ bake is planned, which may help

T07 Superconducting RF 
Table 1: HTC and full injector module parameters.

\begin{tabular}{|l|c|c|}
\hline Parameter & HTC & Injector \\
\hline Number of 2-cell cavities & 1 & 5 \\
Number of HOM loads & 2 & 6 \\
Total 2K load & $\approx 6 \mathrm{~W}$ & $\approx 26 \mathrm{~W}$ \\
Total 5K load & $\approx 12 \mathrm{~W}$ & $\approx 60 \mathrm{~W}$ \\
Total 80K load & $\approx 50 \mathrm{~W}$ & $\approx 700 \mathrm{~W}$ \\
Overall length & $2.6 \mathrm{~m}$ & $5.0 \mathrm{~m}$ \\
\hline
\end{tabular}

with coupler processing as observed during prototype coupler test. Insight gained from the HTC assembly has been applied to the full injector module design to reduce cost and simplify module assembly further. Overall, the choice of building a single-cavity test module first has been highly beneficial.
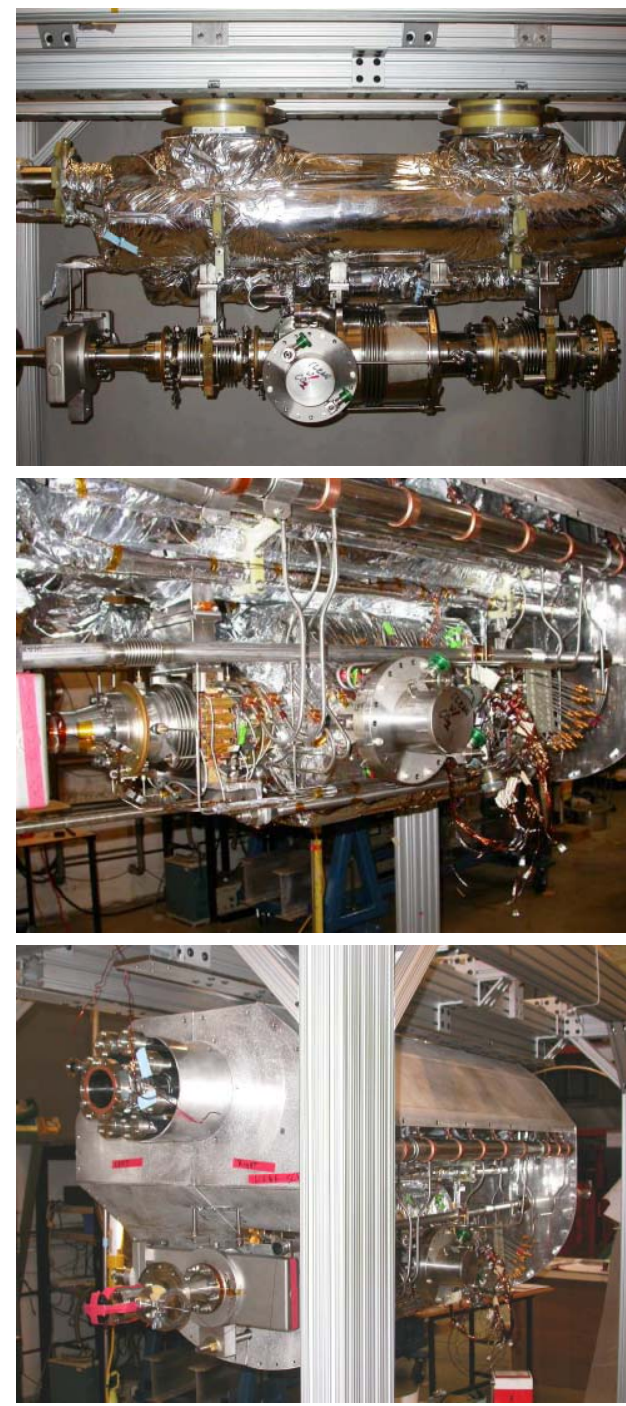

Figure 3: HTC module assembly. Top: Beam line string supported by HGRP. Middle: After adding of $5 \mathrm{~K}$ and $80 \mathrm{~K}$ tubing. Bottom: Nearly closed $80 \mathrm{~K}$ shield.

\section{TEST PLANS AND FUTURE WORK}

First cool down of the horizontal test module is planned for July 2007. This extensive proof-of-principle test will include test and characterization of the cryomodule concept, a full cavity and input coupler test, some HOM damping measurements without beam, as well as a frequency tuner test with first studies on microphonics compensation. The cavity string for the full ERL injector module is presently under assembly. The design and modeling of this module has been finished and all components of the cryovessel have been ordered and are presently under fabrication. We expect to finish the assembly of injector module by end of 2007 with first cool down early 2008 .

\section{SUMMARY}

The assembly of the ERL horizontal test cryomodule is nearly finished. No major design problems have been found during assembly. The design has proven to allow for a fast and easy assembly and alignment. Part of the experience gained during the first module assembly has already been applied to the design of the full injector cryomodule to further reduce cost and ease module assembly. A first extensive test of the HTC is scheduled for July 2007.

\section{REFERENCES}

[1] G. Hoffstaetter et al., this conference, paper MOOBAB02.

[2] D. H. Bilderback, Energy Recovery Linac Experimental Challenges; Proceeding of Future Light Sources 2006 meeting in Hamburg, invited paper PLT03, p1-6 (2006).

[3] G. Hoffstaetter et al., The Cornell ERL Prototype Project, Proceedings of the 2003 Particle Accelerator Conference, Portland, Or, USA (2003).

[4] M. Liepe et al., Design of the CW Cornell ERL Injector Cryomodule, Proceedings of the 2005 particle Accelerator Conference, Knoxvill, TN, USA (2005).

[5] V. Shemelin et al., Dipole-Mode-Free and Kick-Free 2-Cell Cavity for the SC ERL Injector, Proceedings of the 2003 Particle Accelerator Conference, Portland, Or, USA (2003).

[6] Rong-Li Geng et al., this conference, paper WEPMS007.

[7] V. Shemelin et al., Status of the HOM load for the Cornell ERL Injector, Proceedings of EPAC 2006, Edinburgh, Scotland (2006).

[8] V. Shemelin, M. Liepe, H. Padamsee, Characterization of Ferrites at Low Temperature and High Frequency, NIM A 557 (2006) 268-271.

[9] Vadim Veshcherevich et al., this conference (PAC2007), paper WEPMS013.

[10] C. Pagani, SRF Activities at INFN Milano - Lasa, Proceedings of the 10th Workshop of RF Superconductivity, Tsukuba, Japan (2001).

[11] C. Pagani et al.,Further Improvements of the TESLA Test Facility Cryostat in View of the TESLA Collider, Advances in Cryogenic Engineering, Vol. 45A, Edited by Q-S. Shu, Plenum Press, New York (1999).

[12] Peter Quigley et al., this conference, paper MOPAS043. 